\title{
Simulation of Long-Term Destruction of Typical Structural Elements Influenced By an Active Medium and Unsteady Loading
}

\author{
Leonid Fomin ${ }^{1,2}$, Yulia Fomina ${ }^{3}$ \\ ${ }^{1}$ Lomonosov Moscow State University. Research Institute of Mechanics \\ 119192 1, Michurinsky pr., Moscow, Russian Federation \\ fleonid1975@mail.ru; \\ ${ }^{2}$ Samara State Technical University. Department of Applied Mathematics and Computer Science \\ 443100 144, Molodogvardejskaya st., Samara, Russian Federation \\ fleonid1975@mail.ru; \\ ${ }^{3}$ Lyceum 1 \\ 142100 2/24 Serpukhovskaya B, Podolsk, Russian Federation \\ dudorova78@gmail.com
}

\begin{abstract}
The long-term destruction of the shell and plate during creep in the active medium under the conditions of an unsteady complex stress state is investigated. The diffusion process of penetration of the active medium into the material is considered. The influence of the medium on the time to fracture is taken into account by introducing a function of the integral average concentration of the medium into the constitutive and kinetic linear fractional relationships. Comparison of the times to failure using the scalar and vector damage parameters is carried out. The features of using a linear fractional model to describe long-term fracture processes are determined.
\end{abstract}

Keywords: Cylindrical shell, plate, linear fractional model, active medium, unsteady loading, delayed fracture, scalar damage parameter, vector damage parameter

\section{Introduction}

The relevance of solving issues of safe operation of materials and structural elements is undoubted. The most often critical structural elements during long-term operation are subject to a number of destructive factors, among them the following should be noted: high temperature and active working and / or environment in combination with prolonged exposure to external loads. Typical structural elements such as shell and plate are exposed to such influences, for example. The influence of the active medium can be determined both by the diffusion penetration of its elements into the material, and by the corrosive effect, which is inherent in active chemical processes of interaction of working and / or environment with materials of structural elements. Of particular importance is the study of such processes during high-temperature longterm loading of metallic materials and structures under creep conditions [1-7].

This article discusses the diffusion effect of the active medium on the shell and plate, which are made of metallic materials. The specified typical elements are under conditions of non-stationary long-term loading when exposed to high temperatures, which cause creep processes in the metal material. Given the accumulation of damage, such processes over time lead to the destruction of structural elements.

Research in these areas is relevant and has both fundamental and applied nature in the calculation of structural elements for power and aerospace purposes.

\section{Linear Fractional Creep Model}

To describe the creep of metals and alloys in the one-dimensional case, the most widespread is the power-law dependence of the steady-state creep rate $\dot{p}$ on stress $\sigma$, which has the form:

$$
\dot{p}=\alpha \sigma^{n} \quad \alpha=\text { const }
$$


It fairly well approximates the experimental data in the selected narrow stress range for a wide class of metallic materials and alloys, but, as noted by Yu.N. Rabotnov [1], if we take a sufficiently wide range of mechanical stress, then the exponent $n$ becomes stress $\sigma$ dependent.

One of the possible variants of relations for describing creep processes is a linear fractional dependence [8], which in some cases allows one to express the creep rate as a function of stress in a wide range of stresses rather well. S.A. Shesterikov and M.A. Yumasheva was proposed to use such dependence in the following form:

$$
\dot{p}=\beta \frac{\sigma-\sigma_{0}}{\sigma_{b}-\sigma} \quad \sigma>0 \quad \beta=\text { const }
$$

where $\sigma_{0}$ is the creep limit (with $\sigma<\sigma_{0}$ practically no creep deformation), in many cases it can be taken $\sigma_{0}=0, \sigma_{b}$ is the short-term ultimate stress limit at the corresponding test temperature.

At stresses close to $\sigma_{b}$, the creep strain rate $\dot{p}$ increases significantly, and fracture occurs at the times under consideration. Thus, in the specified linear fractional relationship, the stress $\sigma_{b}$ plays the role of the limiting stress. In contrast to the standard power-law dependence, where the stress can be chosen arbitrarily large, in this singular dependence the stress is limited by the value $\sigma_{b}$, which is more justified from the physical and mechanical points of view.

Qualitative graph of the dependence proposed by S.A. Shesterikov and M.A. Yumasheva, when $\sigma_{0}=0$ shown in Fig. 1.

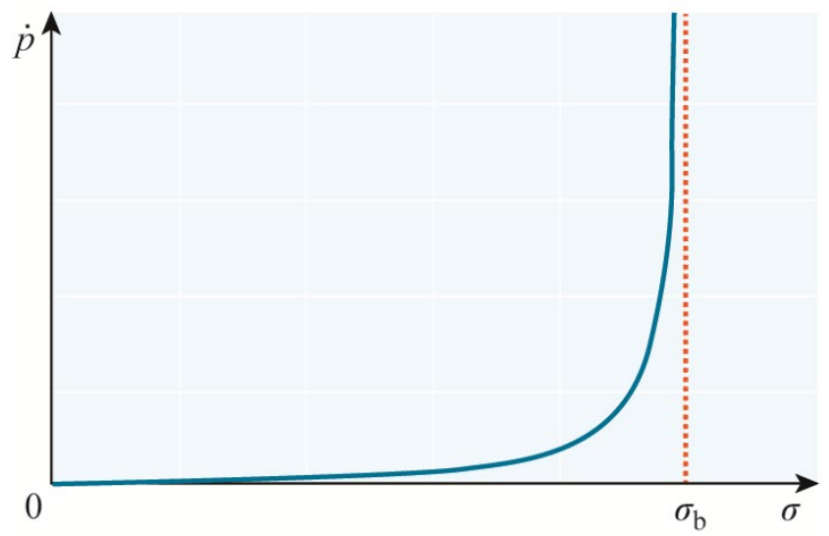

Fig. 1: Linear fractional dependence of the creep strain rate on stress at $\sigma_{0}=0$.

\section{Diffusion of an Active Medium. An Approximate Method for Solving the Diffusion Equation}

To analyze the process of diffusion of the active medium into the material, an approximate method is proposed for solving the diffusion equation based on the introduction of a diffusion front propagating from the surface of a plate or shell (hereinafter, the plate and the shell are designated as a typical element) $[2,5,6]$. This approach allows one to divide the entire cross section of a typical element into perturbed (where the medium has already penetrated into the material) and unperturbed regions (where there is no penetration of the medium yet) and then track the movement of the boundary between these regions in time. The time dependence of the concentration of the active medium in the material of a typical element is approximated in the form of a polynomial, and the boundary and initial conditions are satisfied exactly, and the diffusion equation is satisfied integrally over the entire cross section of a typical element.

In the future, to analyze the effect of an active medium on the time until the destruction of a typical element, a dimensionless integral average concentration $\bar{c}_{m}(\bar{t})$ will be used, which is obtained using the indicated approximate method and has the form: 


$$
\bar{c}_{m}(\bar{t})=\int_{0}^{1} \bar{c}(\bar{z}, \bar{t}) d z=\left\{\begin{array}{lr}
(1 / 3) \sqrt{\bar{t}} & \text { at } \quad 0<\bar{t} \leq 1 \\
1-(2 / 3) \cdot \exp [-(1 / 4)(\bar{t}-1)] & \text { at } \bar{t}>1
\end{array}\right.
$$

Dimensionless variables are introduced here

$$
\bar{z}=2 z / H \quad \bar{t}=48 D t / H^{2} \quad \bar{c}=c / c_{0},
$$

where $z$ is the coordinate along the thickness of the typical wall ( $z=0$ - on the median line of the cross section of the plate or shell, $z>0$ - on the convex side of the plate), $t$ is the time, $c_{0}$ is the constant concentration at the boundary of the material of a typical element and the external active medium, $D=$ const is the diffusion coefficient.

The monograph [2] shows the results of a numerical experiment, which shows a comparison of the corresponding concentrations of the active medium $\bar{c}(\bar{x}, \bar{t})$ and the integral average concentrations $\bar{c}_{m}(\bar{t})$ obtained as a result of the exact (expressed in series) [9] and approximate solutions according to the proposed method, taking into account the introduction of a diffusion front. The closeness of the approximate solution to the exact one is less than 5 percent, depending on the choice of the degree of the polynomial of the desired approximate solution.

Taking into account the fact that the experimental creep curves and times to fracture have, as a rule, a significant scatter at the same mechanical stresses and temperatures, the effect of a slight deviation of the integral average concentration of the active medium on creep and long-term strength in accordance with an approximate solution in comparison with the exact solution is perfectly acceptable.

\section{Cylindrical Shell at an Unsteady Complex Stress State. Statement of the Problem}

Consider a long thin-walled cylindrical shell without bottoms with a radius $R$ of the middle surface and a wall thickness $H$. Let this shell be under the action of unsteady tensile axial force $P$ and internal pressure $Q$. The shell is in an active medium. The effect of the active medium is determined by the diffusion penetration of its elements into the material. A one-dimensional diffusion process is considered. The curvature of the thin-walled shell is small, and the ratio of the shell wall thickness to its radius is much less than unity. This fact makes it possible to treat with a high degree of accuracy the diffusion of the medium into the shell wall as diffusion into a flat plate.

The task is statically definable, and the stress state is determined only by external loads. The stress state in the shell is flat (with zero radial stress $\sigma_{r r}$ ) and homogeneous (the stress state is the same and does not depend on spatial coordinates).

\subsection{Linear Fractional Creep Model. Consideration of the Influence of the Active Medium. Determination the Components of the Stress-Strain State}

The stress tensor components are obtained from the equations of equilibrium of the elementary volume of the solid (equations of the theory of shells [10]):

$$
\sigma_{z z}=P /(2 \pi R H), \quad \sigma_{\theta \theta}=R Q / H
$$

The effect of the active medium was taken into account by introducing into the linear fractional $[6,8]$ constitutive equation of the function of the integral average concentration of the active medium $f\left(\bar{c}_{m}(\bar{t})\right)$ in the shell material.

$$
\dot{p}_{u}=A\left(\sigma_{u} /\left(\sigma_{b}-\sigma_{u}\right)\right) \cdot f\left(\bar{c}_{m}(\bar{t})\right)
$$

where $\dot{p}_{u}$ is the intensity of the creep strain rates, here and below the dot above the corresponding symbol means the time $\bar{t}$ derivative, $\sigma_{u}$ is the stress intensity, $A$ is the material constant.

In further calculations, we will use the linear form of the function $f\left(\bar{c}_{m}(\bar{t})\right)$ :

$$
f\left(\bar{c}_{m}(\bar{t})\right)=1+a \cdot \bar{c}_{m}(\bar{t}), \quad f(0)=1,
$$

where $a=$ const is a constant determined from experiments on long-term strength taking into account the influence of the surrounding active medium $[4,11]$. 
Let us accept the following hypotheses: deformations are small, material incompressibility, elastoplastic deformations are neglected in comparison with creep deformations.

Taking into account the hypothesis of proportionality of stress deviators and creep strain rates for an incompressible materials of solids [1,2], we obtain expressions for the components of axial $\dot{p}_{z z}$ and circumferential $\dot{p}_{\theta \theta}$ creep strain rates:

$$
\dot{p}_{z z}=\frac{A f\left(\bar{c}_{m}(\bar{t})\right)}{\left(\sigma_{b}-\sigma_{u}\right)}\left(\sigma_{z z}-\frac{1}{2} \sigma_{\theta \theta}\right), \quad \dot{p}_{\theta \theta}=\frac{A f\left(\bar{c}_{m}(\bar{t})\right)}{\left(\sigma_{b}-\sigma_{u}\right)}\left(\sigma_{\theta \theta}-\frac{1}{2} \sigma_{z z}\right)
$$

As a result, relations (2) and (5) determine the stress-strain state of a cylindrical shell, taking into account the use of a linear fractional creep model.

\subsection{Linear Fractional Model of Long-Term Destruction with Account the Influence of the Active Medium. Scalar and Vector Parameters of Damage. Criteria of Fracture}

To determine the time to fracture of the shell, we use the kinetic theory of creep and long-term strength by academician Yu.N. Rabotnov [1] and introduce into the kinetic equations a function of the integral mean concentration level $\bar{c}_{m}(\bar{t})$. Consider the kinetic equation taking into account the scalar damage parameter in the following form [6]:

$$
\dot{\omega}=d \omega / d \bar{t}=C\left(\sigma_{u} /\left(\sigma_{b}-\sigma_{u}\right)\right) \cdot f\left(\bar{c}_{m}(\bar{t})\right), \quad \omega(\bar{t}=0)=0
$$

The criterion of fracture is the achievement of the value of the damage parameter equal to unity $\omega^{*}\left(\bar{t}=\bar{t}_{\omega}^{*}\right)=1$ at the moment of time $\bar{t}=\bar{t}_{\omega}^{*}$.

As a variant of the development of simulation of damage accumulation under the condition of long-term hightemperature loading, one can take the vector $\Omega\left(\Omega_{1}, \Omega_{2}, \Omega_{3}\right)[12,13]$ as the damage parameter, the components of which are related to the space of principal stresses $\sigma_{i}(i=1,2,3)$.

In this work, the axes of the introduced cylindrical coordinate system $(z, \theta, r)$ are the principal axes of the stressstrain state. Since the stress state in the considered shell is flat and homogeneous, the kinetic equations taking into account the vector parameter of damage $\boldsymbol{\Omega}[12,13]$ will be taken in the following form [6]):

$$
\dot{\Omega}_{i}=d \Omega_{i} / d \bar{t}=\left\{\begin{array}{ll}
C \cdot\left(\sigma_{i} /\left(\sigma_{b}-\sigma_{i}\right)\right) \cdot f\left(\bar{c}_{m}(\bar{t})\right) & \text { at } \sigma_{i}>0 \\
0 & \text { at } \quad \sigma_{i} \leq 0
\end{array} \quad i=z, \theta\right.
$$

where $\Omega_{i}$ is the projection of the damage vector on to the $i$ axis of the coordinate system. The criterion of fracture is the achievement of the long $\Omega$ vector $\Omega$ of a value equal to unit:

$$
\Omega=\sqrt{\left(\Omega_{z}\right)^{2}+\left(\Omega_{\theta}\right)^{2}} \quad \Omega(\bar{t}=0)=0 \quad \Omega^{*}=\Omega\left(\bar{t}=\bar{t}_{\Omega}^{*}\right)=1
$$

At present, there is an insufficient amount of systematic experimental data on creep deformations and long-term strength under arbitrary unsteady complex stress states. The design and implementation of such experiments is, as a rule, a rather difficult task. An analytical review of such works known to the author of the article is given in [14]. It should also be noted that the random nature of the accumulated damage when the type of complex stress state changes.

The question arises as to how to take into account the accumulation of damage in the process of creep. The scalar damage parameter can be used adequately only in a number of cases. To determine the components of the tensor parameter of damage, it is necessary to search for a sufficiently large number of material constants and functions, carrying out complex setup experiments. According to the author of the article, for modeling the long-term destruction processes, it is optimal to use the vector parameter of damage accumulation.

It should also be noted that the vector approach makes it possible to simulate long-term strength and determine the times to fracture both with a constant position of the main axes and with a change in the orientation of the main axes of the stress-strain state, and even when such a change in the orientation of the main axes is repeated many times. So, for example, in $[15,16]$, the results of known tests of tubular specimens at constant axial stress and constant or alternating 
shear stress are considered. Experiments show that a cyclic change in the sign of shear stresses leads to a significant increase in the time to fracture. To describe this effect, a kinetic approach was used $[12,17]$ with a vector parameter of damage. The application of this approach leads to good agreement between the experimental and theoretical values of the times to fracture

In this paper, we consider two programs of unsteady loading of the shell: separate and combined in time.

Separate program. The following loading program is adopted. First, internal pressure $Q>0$ is applied to the long cylindrical shell during time $0<\bar{t} \leq \bar{t}_{1}$, it is assumed that $\bar{t}_{1}=0.5 \bar{t}_{1}{ }^{*}$ (where $\bar{t}_{1}^{*}$ is the time to fracture of the shell under consideration under the influence of this internal pressure $Q$ only), while the axial tensile force $P=0$. Then during time $\overline{t_{1}}<\bar{t}<\bar{t}^{*}$ (where $\bar{t}^{*}$ - time to fracture of the shell at the end of the loading program) the shell under consideration is subjected to the axial tensile force $P>0$, while the internal pressure is zero (i.e. the conditions $Q=0, P>0$ are hold).

Program combined in time. The combined program differs in that the axial force $P>0$ acts at both stages of loading, while the effect of internal pressure is similar in stages as in the separate loading program. Additionally, the condition $P=2 \pi R^{2} Q$ is accepted.

\subsection{On Determining the Parameters of the Model Equations}

In describing the creep process for a wide class of metallic materials, power-law and fractional-linear creep models are used, which are indicated at the beginning of Section 2 of this article. Material parameters $\alpha, n, \beta$ are determined based on the processing of the experiment. An experiment is a tensile creep test of a standard sample made of a material whose properties are to be studied. As a result of the experiment, a family of creep curves is obtained, each of which is obtained at a certain mechanical stress $\sigma$. To determine, the second sections of the family of creep curves are used - the sections of steady-state creep on each curve of the family. The short-term strength limit $\sigma_{b}$ at an appropriate temperature is determined by a known standard method. The values of the material constants will generally depend on the particular material selected and the test temperature.

The parameter in equation (3) is determined in a similar way, while the experiment is carried out in a neutral medium. The effect of the active medium in equation (3) is taken into account by introducing a function $f\left(\bar{c}_{m}(\bar{t})\right)=1+a \cdot \bar{c}_{m}(\bar{t})$ into the specified equation, the definition of the constant $a$ is described in detail in [4]. For example, when determining the long-term strength of samples made of low-carbon steel, taking into account the diffusion of oxygen into the samples from the active medium, the value $a=9.5$ was obtained.

The characteristic $C$ of the material (in equation (6)) is determined on the basis of equating the values of the theoretical and experimental times to fracture in the creep test of a standard sample in a neutral medium. The theoretical

time to failure $\bar{t}_{\omega}^{*}$ is determined by integrating equation (6) to the damage $\omega$ value to the value $\omega^{*}=\omega\left(\bar{t}=\bar{t}_{\omega}^{*}\right)=1$, in this calculation, the value of $f\left(\bar{c}_{m}(\bar{t})\right)=1$ is taken to be equal to one.

It should also be noted that to solve the model problem, some model values of the specified material parameters can be taken.

\subsection{Analysis of the Obtained Results}

As a result of the calculation, dimensionless times to fracture were obtained for separate and combined loading programs using the scalar damage parameter $\bar{t}_{\omega}^{*}=0.614$ and using the vector damage parameter $\bar{t}_{\Omega}^{*}=0.817$. The ratio of times to fracture is $\xi=\bar{t}_{\Omega}^{*} / \bar{t}_{\omega}^{*}=1.33$. Thus, the time to fracture when using the vector damage parameter is greater than the time to failure obtained when using the scalar damage parameter.

Under the selected condition $P=2 \pi R^{2} Q$, the times until the destruction of the cylindrical shell in separate and combined loading programs are the same. This is true for both scalar and vector damage parameters. With such 
$P=2 \pi R^{2} Q$ a dependent on each other setting of external loads, dimensionless parameters $\bar{C}=\left(\sigma_{b} H-R Q\right) /(R Q)$ and $\bar{P}=P /\left(2 \pi R H \sigma_{b}-P\right)$ must satisfy the condition $\bar{C} \bar{P}=1$. When the external loads under consideration are set independently, the dimensionless parameters $\bar{C}$ and $\bar{P}$ are set independently of each other.

\section{Plate At An Unsteady Complex Stress State With Account The Influence Of The Active Medium. Determination the Time to Fracture}

Similar hypotheses and approaches (linear fractional model, an approximate method for solving the diffusion equation, scalar and vector parameters of damage) were applied to study the long-term destruction of a plate in an active medium. In accordance with the formulation of the problem, the plate is in a non-stationary complex stress state under the piecewise constant action of bending moments $M_{1}$ and $M_{2}$, distributed along the mutually orthogonal edges of the plate [6].

The table 1 shows the results of calculating the times to fracture $\bar{t}_{\omega}^{*}$ and ${\overline{t_{\Omega}}}_{\Omega}^{*}$ using the scalar and vector damage parameters, respectively, for different values $\zeta=M_{2} / M_{1}$.

Table 1: Failure times to fracture for different values $\zeta$.

\begin{tabular}{|c|c|c|}
\hline$\zeta=M_{2} / M_{1}$ & $\bar{t}_{\omega}^{*}$ & $\bar{t}_{\Omega}^{*}$ \\
\hline 1.5 & 0.107 & 0.123 \\
\hline 1.0 & 0.160 & 0.205 \\
\hline 0.5 & 0.292 & 0.407 \\
\hline
\end{tabular}

In fig. 2 shows the dependences of damage on time at the values $\zeta=0.5,1,1.5$ obtained for the scalar $\omega(\bar{t})$ (solid curves) and vector $\Omega(\bar{t})$ (dashed curves) damage parameters.

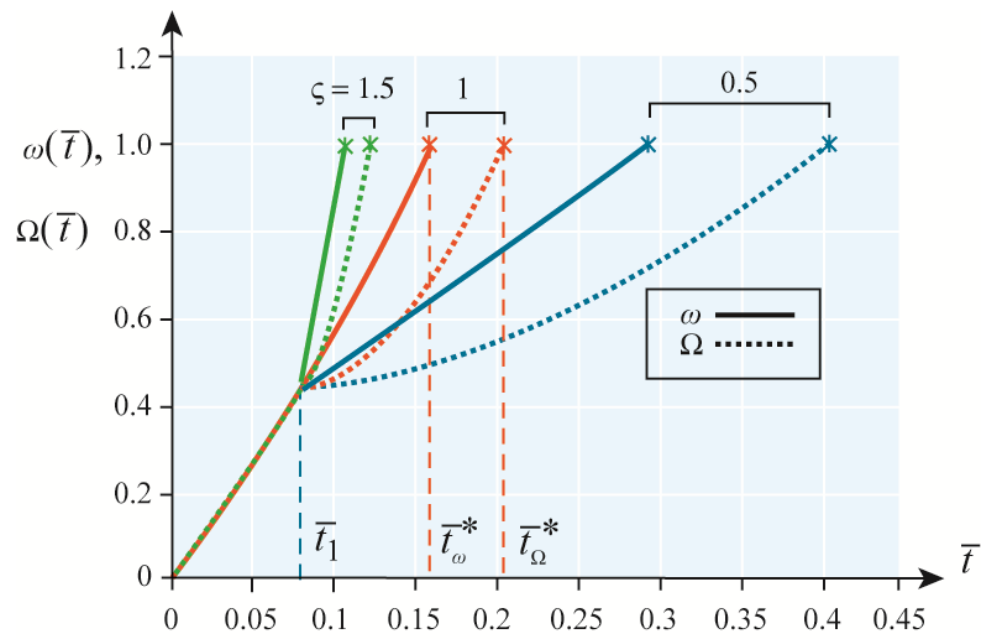

Fig. 2: Damage versus time for scalar and vector approaches. 
In the time interval $0<\bar{t}<\overline{t_{1}}$ (as a result of the calculation, the value $\overline{t_{1}}=0.08$ was obtained), the graphs for damage obtained using the scalar and vector approaches coincide.

\section{Conclusion}

Comparison of times to fracture of typical structural elements (shell and plate) is carried out using scalar and vector parameters of damage. The time to failure when using the vector damage parameter $\bar{t}_{\Omega}^{*}$ is greater than the time to failure obtained when using the scalar damage parameter $\bar{t}_{\omega}^{*}$. The ratio of times until the destruction of the shell is $\xi=\bar{t}_{\Omega}^{*} / \bar{t}_{\omega}^{*}=1.33$.

The conducted research shows the difference in the mechanisms of damage accumulation during prolonged hightemperature loading. The result obtained confirms similar results obtained by the authors of [6] and shown in Section 5 of this article in the study of long-term unsteady loading of a plate in mutually orthogonal planes. In the specified work [6], the ratios of times to fracture $\bar{t}_{\Omega}^{*} / \bar{t}_{\omega}^{*}$, depending on the ratio of the values of bending moments, are in the range $\bar{t}_{\Omega}^{*} / \bar{t}_{\omega}^{*}=1.15-1.39$.

This research has both a fundamental character and an applied aspect. The proposed approaches and research results can be applied in the energy, chemical and aerospace industries.

\section{Acknowledgements}

The author of this article is grateful to Professor A.M. Lokoshchenko for attention to the work done and useful discussions.

This study was supported by the Russian Science Foundation (RSF 19-19-00062, Samara State Technical University).

\section{References}

[1] Yu. Rabotnov, Creep Problems in Structural Members. Amsterdam. Noth-Holland, 1969.

[2] A. Lokoshchenko, Creep and Long-Term Strength of Metals. London. New York: CISP. CRC Press. Taylor Francis Group. Boca. Raton, 2018.

[3] A. M. .Lokoshchenko and L. V. Fomin, "Modeling the behavior of materials and structural elements exposed to aggressive media (review)," Strength Plast. Prob., vol. 80, no. 2, pp. 145-179, 2018.

[4] L. V. Fomin, "Description of the long-term strength of stretchable rods of rectangular and round cross-sections in high-temperature air,” J. Samara State Tech. Univ. Ser. Phys. Math. Sci., vol. 32, no. 3, pp. 87-97, 2013.

[5] L. V. Fomin, "Steady-state creep of a composite rod in tension in the presence of an aggressive environment," Mech. Compos. Mater., vol. 52, no. 6, pp. 741-750, 2017.

[6] A. M. Lokoshchenko and L. V. Fomin, "Delayed fracture of plates under creep condition in unsteady complex stress state in the presence of aggressive medium," Applied Mathematical Modelling. vol. 60, pp. 478-489, 2018

[7] L. V. Fomin, Yu. G. Basalov and A.M. Lokoshchenko, "On taking into account the influence of corrosion processes on the long-term fracture of a rod under creep conditions" Mech. Compos. Mater. Struct, vol. 25, no. 3, pp. 327-335, 2019.

[8] Shesterikov and M.A. Yumasheva, "More precise specification of the equation of state in creep theory," Mech. of Solids, vol. 19, no. 1, pp. 81-85, 1984.

[9] A. Tikhonov and A. Samarskiy, Equations of Mathematical Physics. Ed. 7, 2004.

[10] S. Timoshenko and S. Woinowsky-Krieger, Theory of Plates and Shells: 2th edition. New York: McGraw- Hill, 1959.

[11] I. A. Oding and Z. G. Fridman. "Role of surface layers during delayed fracture of metals under creep condition,". Industrial Laboratory. vol. 25, no. 3, pp. 329-332, 1959.

[12] L. M. Kachanov, Foundations of the Mechanics of Damage. Moscow: Nauka, 1974. 
[13] I. V. Namestnikova and S. A. Shesterikov, "Vector representation of damage parameter. Deformation and destruction of solids," in Proceedings of the Institute of Mechanics of Moscow State University. Moscow: Publishing House of Moscow University, 1985, pp. 43-52.

[14] A. M. Lokoshchenko, L. V. Fomin, W. V. Teraud, Yu. G. Basalov and V. S. Agababyan, "Creep and long-term strength of metals under unsteady complex stress states (Review),“J. Samara State Tech. Univ. Ser. Phys. Math. Sci., vol. 24, no. 2, pp. 275-318, 2020.

[15] A. M. Lokoshchenko, "Use of a vector damage parameter in modeling of long-term strength of metals, " Mech. of Solids, vol. 51, pp. 315-320, 2016.

[16] A. M. Lokoshchenko, "Modeling the long-term strength of metals in an unsteady complex stress state," Mech. of Solids, vol. 53, pp. 88-100, 2018.

[17] L. M. Kachanov, "Fractures under creep conditions under complex loading, "Izv. Academy of Sciences of the USSR. Mekhanika tverdogo tela, no 5, pp. 11-15, 1972. 